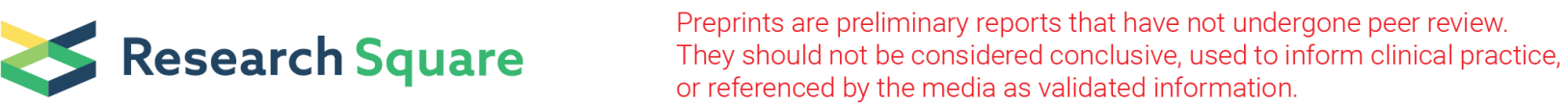

\section{Transcatheter arterial infusion combined with radioactive particles in the treatment of advanced body/tail pancreatic cancer: a retrospective cohort study}

Shilong Han

Shanghai Tenth People's Hospital, Tongji University

Chuanwu Cao

Shanghai Tenth People's Hospital, Tongji University

Yifeng Yuan

Shanghai Tenth People's Hospital

Jun Chen

Shanghai Tenth People's Hospital, Tongji University

Linan Yin

Harbin Medical University Cancer Hospital

Huirong Xu

Shandong Cancer Hospital Affiliated to Shandong University

Jingzhou Liu

Shandong Cancer Hospital Affiliated to Shandong University

Wujie Wang

The Second Hospital of Shandong University

\section{Yuliang Li}

The Second Hospital of Shandong University

\section{Lizhou Wang}

Affiliated Hospital of Guizhou Medical University

Shi Zhou

Affiliated Hospital of Guizhou Medcial University

Fenqiang Li

First Hospital of Lanzhou University

Wenhui Wang

First Hospital of Lanzhou University

Jiansong Ji

Lishui Hospital of Zhejiang University

Haideng Long

Hefei Hospital Affiliated to Anhui Medical University 


\section{Shiwu Yin}

Hefei Hospital Affiliated to Anhui Medical University

Jue Li

School of Medicine, Tongji University

Jianjun Han

Shandong Cancer Hospital Affiliated to Shandong University

\section{Ruibao Liu}

Harbin Finance University Cancer Hospital

Maoquan Li

Shanghai Tenth People's Hospital, Tongji University

Xiaoping Zhang ( $\sim$ zxpkxy@tongji.edu.cn )

Shanghai Tenth People's Hospital, Tongji University

\section{Research article}

Keywords: advanced body/tail pancreatic cancer, transcatheter arterial infusion, radioactive particles

Posted Date: July 21st, 2020

DOI: https://doi.org/10.21203/rs.3.rs-44813/v1

License: (1) This work is licensed under a Creative Commons Attribution 4.0 International License. Read Full License

Version of Record: A version of this preprint was published at Pancreas on August 3rd, 2021. See the published version at https://doi.org/10.1097/MPA.0000000000001851. 


\section{Abstract \\ Background}

Patients with advanced body/tail pancreatic cancer have poor quality of life and low overall survival rate. In recent years, interventional diagnosis and treatment of advanced pancreatic cancer have become increasingly widespread. This retrospective cohort study investigated the efficacy of routine intravenous chemotherapy (the control group), transcatheter arterial infusion (TAl) chemotherapy, and TAl combined with radioactive particles as therapeutic methods for advanced body/tail cancer pancreatic by assessing the short-term and overall survival rates.

\section{Methods}

We screened our prospective database for patients with advanced body/tail pancreatic cancer, which tumor deemed unresectable and no other confirmed malignant tumors, patients were assigned into three groups according to their treatment. Analyses with regard to the clinical responses, the 6,12 , and 18 month survival rates and overall survival rates were performed.

\section{Results}

The median survival time was 6 months in the control group, 10 months in the TAl group and 13 months in the TAI combined group. The Kaplan-Meier estimates of the OS among the three groups, indicating that there is significant difference among three groups $(P \otimes 0.000)$. The clinical remission rates were $17.5 \%$ in the control group, $41.5 \%$ in the TAI group, and $48.0 \%$ in the TAl combined group. Covariates analyzed showed that different treatment methods and times affected the results significantly ( $P \otimes 0.002)$.

\section{Conclusions}

In the treatment of advanced body/tail pancreatic cancer, TAI and TAI combined with radioactive particles significantly improved the clinical outcomes in patients compared with routine intravenous chemotherapy.

\section{Background}

The incidence of pancreatic cancer (PC) has increased considerably over recent decades throughout the world [1]. Pancreatic carcinoma is asymptomatic in the early stages, so patients are often diagnosed with an advanced stage of the disease (TMN stages III and IV [2]), which in the majority of cases is deemed inoperable [3]. Chemotherapeutic regimens and chemoradiotherapy are commonly administered in an attempt to slow disease progression; however, these strategies are often ineffective [4],[5]. Molecular 
targeting and stem cell therapy offer alternative treatment strategies; however, these methods are difficult to apply widely in clinic due to technical and economic constraints.

Regional intra-arterial chemotherapy has also been proposed as a potential treatment option for pancreatic carcinoma [1]. Chen and colleagues employed transcatheter arterial infusion chemotherapy (TAI) involving gemcitabine (GEM) and oxaliplatin (OXA) in the treatment of unresectable pancreatic cancer and found it to be well tolerated and highly effective [6]. It has been suggested that if pancreatic cancer initially diagnosed as unresectable can be controlled and disease progression slowed by effective therapy, surgical resection may become an option, thereby potentially increasing long-term survival [7]. The aim of therapy in the case of this malignancy could therefore be localized control and containment to enable surgery.

The implantation of radioactive particles combined with chemotherapy or radiotherapy is another technique that has been reported to be effective for the local control of pancreatic cancer [8], [9]. I $^{125}$ particles are the most commonly used radioactive particles for this type of cancer therapy because their long half-life and short radiation distance mean that high doses can be targeted specifically to the tumor, thereby not damaging the surrounding tissue [10]. A study by Yu and colleagues showed that when chemoradiotherapy combined with $\mathrm{I}^{125}$ implantation was used to treat advanced pancreatic cancer, a higher local control rate and a better quality of life were recorded in patients compared with the use of chemoradiotherapy alone [11].

In this study, we compared a range of treatments for advanced body/tail pancreatic cancer involving intravascular interventional therapy and TAI combined with particle therapy in terms of the short-term and overall survival rates. Our findings highlight the potential of these novel treatment strategies to significantly improve the survival of patients with advanced pancreatic cancer.

\section{Methods}

\section{Patients}

In total, 360 patients with locally advanced body/tail pancreatic cancer were reviewed in 8 centers, in which 237 patients diagnosed met the inclusion criterion. Diagnoses were confirmed by histological examination and either clinical or radiological findings. The inclusion criteria for the study were as follows: (1) pathologically or radiologically-diagnosed pancreatic cancer in the body/tail ; (2) tumor deemed unresectable due to the advanced stage of the disease as determined by TNM staging according to AJCC [2], which included stage $₫ A$ and beyond; (3) No other confirmed malignant tumors. The exclusion criteria were as follows: (1) patients with mental disorders or with severe cardiopulmonary dysfunction or advanced cachexia, (2) Multiple metastases throughout the body, (3) insufficient coagulation (platelets $<30 \times 10 /$ L).

\section{Classification}


Patients were assigned to three groups according to their treatment: group A, routine intravenous chemotherapy; group B, transcatheter arterial infusion (TAI) chemotherapy; group C, TAl combined with radioactive particles as therapeutic.

\section{Routine intravenous chemotherapy}

For the control group, concurrent chemotherapy was performed. This involved the intravenous administration of GEM $800-1000 \mathrm{mg} / \mathrm{m}^{2}$, no less than $30 \mathrm{~min}$, once a week for 2 weeks, breaking for 1 week, and starting for another course of treatment, totally four courses.

\section{Transcatheter arterial infusion (TAl) chemotherapy}

For the TAl treatment group, a 5-Fr arterial sheath was placed in the right femoral artery under local anesthesia and the celiac trunk was selected by 5-Fr RH catheter under digital subtraction angiography (DSA). Then, a combination of GEM $800-1000 \mathrm{mg} / \mathrm{m}^{2}$, was injected via the RH catheter for $2 \mathrm{~h}$ at $1 / 2$ dosage, and so was the 5-fluorourcil (FU) $500 \mathrm{mg} / \mathrm{m}^{2}$ but for $20 \mathrm{~h}$.

\section{Radioactive $\mathrm{I}^{125}$ particle implantation}

Patients in the TAl combined treatment group received arterial infusion combined with ablation or radioactive particles. For radioactive $\mathrm{I}^{125}$ particle implantation in the TAl combined treatment group, patients first underwent CT (uMI-510 PET-CT, United Imaging, Shanghai, China), and the images were entered into a treatment plan system (TPS, Astro Tech Ltd, Beijing, China). The gross tumor volume (GTV) was confirmed by analysis of the scans by a medical specialist, and $0.5-1.0 \mathrm{~cm}$ was added to the boundary of the GTV to include the periphery of the tumor. Then, implantation of $\mathrm{I}^{125}$ radiological particles (radioactivity, 0.55-0.75 mCi; dose, 120-140Gy; dose rate, 0.05-0.10Gy h21; half-life, 59.6 days; effective radius, $1.72 \mathrm{~cm}$; Zhibo Bio-Tech Ltd, Beijing, China) was performed at the periphery of the tumors. The radioactive dose was evaluated 1 week after implantation, and a D90 (the dose to $90 \%$ of the volume) of 60-140 Gy was expected. If the actual dose was lower than this, implantation could be repeated.

\section{Therapeutic effect}

Changes in imaging and OS, as well as 6, 12, 18 -month survival rates, were used as indicators of efficacy of the treatment. The treatment response was evaluated 4-6 weeks after each treatment using the modified Response Evaluation Criteria in Solid Tumors (mRECIST).

A complete response (CR) was defined as disappearance of any intratumoral arterial enhancement in all lesions; a partial response (PR) was defined as a 30\% decrease in the sum of the diameters of viable (contrast enhancement in the arterial phase) lesions; progressive disease (PD) was defined as an increase of $20 \%$ in the sum of the diameters of viable lesions; and stable disease (SD) was defined as any case that did not qualify as either PR or PD. The two largest foci were selected among the multifocal 
lesions for measurement. CR and PR were considered as valid. OS refers to the time starting at initial interventional therapy until death or final follow-up (December 2015).

\section{Follow-up}

Follow-up of the patients was conducted from January, 2009, to December, 2015, to assess patient survival. Of the 237 patients enrolled in the study, 9 cases were lost at follow-up, constituting a loss rate of $3.80 \%$.

\section{Statistical analysis}

Data were analyzed using the SPSS ${ }^{\circledR}$ version 16.0 software. OS, $6,12,18$-month survival rates in the three groups were compared by analysis of variance (ANOVA). OS rates were also estimated by the Kaplan-Meier method, and survival differences were analyzed by the log-rank test. $P<0.05$ was considered statistically significant.

\section{Results}

The baseline characteristics of these patients are presented in Table 1. The study group comprised 134 male and 103 female patients, with an average age of $65.89 \pm 11.64$ years (range: $30-90$ years). The control group $(n=80)$ who received routine intravenous chemotherapy, The TAl group $(n=82)$ who received arterial infusion, and the TAl combined group $(n=75)$ who received arterial infusion combined with radioactive particle implantation. 
Table 1

Patients' baseline characteristics

\begin{tabular}{|c|c|c|c|c|}
\hline Variables & $\begin{array}{l}\text { Control group } \\
n=80\end{array}$ & $\begin{array}{l}\text { TAl group } \\
\mathrm{n}=\mathbf{8 2}\end{array}$ & $\begin{array}{l}\text { TAl combined group } \\
\mathrm{n}=75\end{array}$ & $P$ value \\
\hline Gender & & & & 0.090 \\
\hline Male & $53(66.25 \%)$ & $41(50 \%)$ & $40(53.33 \%)$ & \\
\hline Female & 27 (33.75\%) & $41(50 \%)$ & 35 (46.67\%) & \\
\hline Age (years) & $66.31 \pm 11.76$ & $68.11 \pm 13.08$ & $66.84 \pm 13.17$ & 0.167 \\
\hline TNM stage & & & & 0.679 \\
\hline T3N1M0 & $10(12.5 \%)$ & 9 (10.96\%) & $7(9.3 \%)$ & \\
\hline T3N1M1 & 62 (87.5\%) & 73 (89.2\%) & 68 (90.67\%) & \\
\hline Tumor size & & & & 0.202 \\
\hline$<3.0$ & $1(1.25 \%)$ & $0(0 \%)$ & $0(0 \%)$ & \\
\hline $3.0-5.0$ & $2(2.5 \%)$ & $0(0 \%)$ & $0(0 \%)$ & \\
\hline$>5.0$ & 77 (96.25\%) & 82 (100\%) & 75 (100\%) & \\
\hline Metastasis & & & & 0.685 \\
\hline Yes & 75 (93.75\%) & 74 (90.24\%) & 68 (90.67\%) & \\
\hline No & 5 (6.25\%) & $8(9.76 \%)$ & $7(9.3 \%)$ & \\
\hline Ascites & & & & 0.337 \\
\hline Yes & $6(7.5 \%)$ & $2(2.4 \%)$ & $4(5.3 \%)$ & \\
\hline No & 74 (92.5\%) & $80(97.6 \%)$ & $71(94.7 \%)$ & \\
\hline Jaundice & & & & 0.000 \\
\hline Yes & $14(17.5 \%)$ & $42(51.2 \%)$ & 8 (10.7\%) & \\
\hline No & 66 (82.5\%) & $40(48.8 \%)$ & 67 (89.3\%) & \\
\hline Child-Pugh class & & & & 0.000 \\
\hline A & $0(0 \%)$ & $0(0 \%)$ & $0(0 \%)$ & \\
\hline B & $16(20.0 \%)$ & $19(23.2 \%)$ & $40(53.3 \%)$ & \\
\hline C & $64(80.0 \%)$ & $63(76.8 \%)$ & $35(46.7 \%)$ & \\
\hline CA $19-9$ & & & & 0.131 \\
\hline
\end{tabular}




\begin{tabular}{|llll|}
\hline Variables & $\begin{array}{l}\text { Control group } \\
\mathbf{n = 8 0}\end{array}$ & $\begin{array}{l}\text { TAl group } \\
\mathbf{n = 8 2}\end{array}$ & $\begin{array}{l}\text { TAl combined group } \\
\mathbf{n = 7 5}\end{array}$ \\
\hline Normal & $12(15.0 \%)$ & $20(23.4 \%)$ & $21(28.0 \%)$ \\
\hline Unusual & $68(85.0 \%)$ & $62(75.6 \%)$ & $54(72.0 \%)$ \\
\hline CA 19 -9 , carbohydrate antigen $19-9$. & \\
\hline
\end{tabular}

\section{Comparison of the OS times among the three groups}

The median survival time were $6.54 \pm 0.63$ months in the control group, $10.12 \pm 0.80$ months in the TAI group, and $13.62 \pm 0.98$ months in the TAl combined group. Figure 1 illustrates the Kaplan-Meier estimates of the OS times among the three groups, indicating that TAl treatment and TAI combined with radioactive particles therapy significantly prolongs survival times in patients with advanced body/tail pancreatic cancer compared with routine intravenous chemotherapy. The data indicate that, on average, this therapeutic combination can extend the survival times of patients with advanced pancreatic cancer by 3 months compared with TAl alone.

\section{Comparison of the overall response to therapy among the three groups}

The patients in each of the groups were evaluated for their response to therapy. As shown in Table 2, the overall control rates (CR + PR) were improved in the TAl (41.5\%) and TAl combined (48.0\%) treatment groups compared with the control group (17.5\%). Furthermore, the rates of stable and progressive disease were decreased in the two treatment groups (32.9\% and $25.6 \%$ for the TAl group, and $28.0 \%$ and $24.0 \%$ for the TAl combined group, respectively) compared with the control group ( $47.5 \%$ and $35.0 \%$, respectively).

Table 2

Overall response to therapy in the three groups of patients

\begin{tabular}{|llllll|}
\hline Response, $\mathbf{n}(\%)$ & Control group & TAl group & TAl combined group & Total & P value \\
& $\mathbf{n = 8 0}$ & $\mathbf{n = 8 2}$ & $\mathbf{n = 7 5}$ & $\mathbf{n = 2 3 7}$ & \\
\hline CR + PR, $\mathrm{n}(\%)$ & $14(17.5 \%)$ & $34(41.5 \%)$ & $36(48.0 \%)$ & $84(35.4 \%)$ & 0.000 \\
SD, $\mathrm{n}(\%)$ & $38(47.5 \%)$ & $27(32.9 \%)$ & $21(28.0 \%)$ & $86(36.3 \%)$ & \\
PD, $\mathrm{n}(\%)$ & $28(35.0 \%)$ & $21(25.6 \%)$ & $18(24.0 \%)$ & $67(28.3 \%)$ & \\
\hline CR, complete remission; PR, partial remission; SD, stable disease; PD, progressive disease. & \\
\hline
\end{tabular}

To investigate the response to therapy on an individual level, one cases were analyzed in greater depth. Figure 2 provides representative CT images for one particular case studies diagnosed with advanced body/tail pancreatic cancer who received TAl combined with radioactive particles therapy. A 78-year-old man diagnosed with advanced body/tail pancreatic cancer who had previously been treated with a highintensity ultrasonic focusing knife. The cancer was located in the pancreatic body (Fig. 2A, indicated by 
an arrow). After arterial infusion combined with radioactive particles therapy, a partial response was achieved (Fig. 2B, indicated by an arrow). Positive responses to therapy were therefore achieved in the case.

\section{Comparison of the 6,12 , and 18-month survival rates among the three groups}

The 6,12 , and 18-month survival rates were found to be significantly improved in the TAl group (71.95\%, $17.07 \%$, and $7.32 \%$ ) and the TAl combined group (80\%,53.33\%, and $20 \%$ ) compared with the control group $(37.5 \%, 11.25 \%$, and $3.75 \%$, respectively) (Table 3$)$. Taken together, our results provide evidence that TAI, and TAI combined with radioactive particles therapy, can significantly improve the survival of patients with advanced body/tail pancreatic cancer compared with routine intravenous chemotherapy.

Table 3

Comparisons of the 6,12, and 18-month survival rates among the three groups

\begin{tabular}{|llllll|}
\hline Time (months) & & Control group & TAl group & TAl combined group & P value \\
\hline 6 & & Cases, $n(\%)$ & Cases, $n(\%)$ & Cases, $n(\%)$ & \\
\hline & & & & & 0.000 \\
\hline & Survival & $30(37.5 \%)$ & $59(71.95 \%)$ & $60(80 \%)$ & \\
& Death & $50(62.5 \%)$ & $23(28.05 \%)$ & $15(20 \%)$ & 0.000 \\
\hline 12 & & & & \\
\hline & Survival & $9(11.25 \%)$ & $14(17.07 \%)$ & $40(53.33 \%)$ & 0.002 \\
\hline & Death & $71(88.75 \%)$ & $68(82.93 \%)$ & $35(46.67 \%)$ & \\
\hline & Survival & $3(3.75 \%)$ & $6(7.32 \%)$ & $15(20 \%)$ & $60(80 \%)$ \\
\hline
\end{tabular}

\section{Discussion}

Previous studies have shown that ${ }^{125}$ I particle implantation is a feasible and safe procedure for unresectable pancreatic cancer [12]; however, a large-scale study to assess the long-term effects of this combination treatment on patient survival has been lacking. Here, we report a retrospective cohort study investigating the curative effects of TAl chemotherapy with or without radioactive particle implantation in patients with unresectable advanced body/tail pancreatic cancer. The survival was positively affected as demonstrated by the 12 -month survival rate $(53.33 \%$ vs. $11.25 \%$ for the combined treatment vs. control groups) and median survival times (13.62 \pm 0.98 months vs. $6.54 \pm 0.63$ months for the combined treatment vs. control groups). 
In a previous study, Hong and colleagues assessed the clinical effectiveness and safety of continuous TAl of GEM and FU versus systemic venous chemotherapy in patients with advanced pancreatic carcinoma [13]. In our study, TAl chemotherapy involved a combination of GEM $800-1000 \mathrm{mg} / \mathrm{m}^{2}$, and 5FU $500 \mathrm{mg} / \mathrm{m}^{2}$; RFA at a temperature of 85-100; and a dosage of $120-140$ Gy of ${ }^{125} \mathrm{I}$ particles was used for implantation. Several previous studies have also reported improved clinical outcomes in patients with advanced pancreatic cancer after receiving ${ }^{125} \mathrm{I}$ particle implantation combined with chemotherapy or radiotherapy in various combinations at a range of dosages [9],[11], [14],[15],[16]. For example, Yu and colleagues performed ${ }^{125} \mathrm{I}$ particle implantation at a lower dose $(88.71 \mathrm{~Gy})$ combined with chemotherapy (GEM, $1000 \mathrm{mg} / \mathrm{m}^{2}$ ) and radiotherapy (30.62 Gy) and obtained a local control rate of 73.33\%, a 12-month survival rate of $72.0 \%$, and a median overall survival time of 14 months in advanced pancreatic cancer patients [11]. There is therefore accumulating evidence for the value of combining different treatment modalities to control unresectable pancreatic malignancies to extend the life expectancy of patients.

The combined therapeutic use of TAI with radioactive particle implantation has been more widely documented for hepatocellular carcinoma (HCC) [17],[18],[19],[20]. In recent years, the ${ }^{125}$ I particle implantation therapy has gradually been applied clinically and has achieved certain therapeutic effects. A study by Dai F and colleagues showed that the ${ }^{125}$ I particle implantation therapy significantly inhibited the local growth of advanced NSCLC [21]. And a study by Sun and colleagues demonstrated that the combination of transcatheter arterial chemoembolization with endovascular implantation of ${ }^{125}$ I particles was effective at increasing the survival rates in HCC patients with portal vein tumor thrombosis compared with transcatheter arterial chemoembolization treatment alone [20]. Consistently, it was found in our study that the TAl combination therapy significantly improved clinical outcome of patients with unresectable pancreatic cancer.

\section{Conclusions}

the implantation of ${ }^{125}$ p particles into tumors combined with TAl chemotherapy results in an improved response rate and prolonged survival in patients with unresectable advanced body/tail pancreatic cancer compared with standard intravenous chemotherapy. This offers a promising therapeutic strategy with which to extend the lifespan of patients with this type of malignancy.

\section{Declarations}

\section{Ethics approval and consent to participate}

This study was approved by the Ethics Committee of the Shanghai Tenth People's Hospital, Tongji University. Written informed consent and permissions for the possibility of utilizing their clinical data anonymously were obtained from all subjects.

\section{Consent for publication}


Not applicable

\section{Availability of data and materials}

The datasets used and/or analysed during the current study are available from the corresponding author on reasonable request.

\section{Competing interests}

The authors declare that they have no competing interests.

\section{Funding}

This work was supported by the National Natural Science Foundation of China (81801805), the Shanghai Sailing Program (16YF1409000), and the Climbing Talent Program of Shanghai Tenth People's Hospital.

\section{Authors' contributions}

SH, CC, and YY: Substantial contributions to acquisition of data, analysis and interpretation of data, drafting the article, performed the statistical analyses, revising it critically for important intellectual content. JC, LY, HX, JL, WW, and YL: Substantial contributions to acquisition of data, analysis and interpretation of data, and performed the statistical analyses. LW, SZ, FL, WW, JJ, HL, SY, JL, and JH: Substantial contributions to acquisition of data, analysis and interpretation of data. RL, ML, and XZ: Substantial contributions to study conception and design, analysis and interpretation of data. All authors involved in revising it critically for important intellectual content, read and approved the final manuscript.

\section{Acknowledgements}

We thank the statistician Mr. Yongtao Zheng for his correction during the data processing.

\section{References}

1. Ma N, Wang Z, Zhao J, Long J, Xu J, Ren Z, et al. Improved Survival in Patients with Resected Pancreatic Carcinoma Using Postoperative Intensity-Modulated Radiotherapy and Regional IntraArterial Infusion Chemotherapy. Med Sci Monit. 2017;23:2315-23.

2. Edge SB, Compton CC. The American Joint Committee on Cancer: the 7th edition of the AJCC cancer staging manual and the future of TNM. Ann Surg Oncol. 2010;17:1471-4.

3. Datta NR, Pestalozzi B, Clavien PA, Siebenhüner A, Puric E, Khan S, et al. "HEATPAC" - a phase II randomized study of concurrent thermochemoradiotherapy versus chemoradiotherapy alone in locally advanced pancreatic cancer. Radiat Oncol. 2017;12:183.

4. Wanebo HJ, Glicksman AS, Vezeridis MP, Clark J, Tibbetts L, Koness RJ, et al. Preoperative chemotherapy, radiotherapy, and surgical resection of locally advanced pancreatic cancer. Arch Surg. 2000;135:81-7. discussion 88. 
5. Rombouts SJ, Vogel JA, van Santvoort HC, van Lienden KP, van Hillegersberg R, Busch ORC, et al. Systematic review of innovative ablative therapies for the treatment of locally advanced pancreatic cancer. Br J Surg. 2015;102:182-93.

6. Chen Y, Wang XL, Wang JH, Yan ZP, Cheng JM, Gong GQ, et al. Transarterial infusion with gemcitabine and oxaliplatin for the treatment of unresectable pancreatic cancer. Anticancer Drugs. 2014;25:958-63.

7. Doi T, Homma H, Akiyama T, Mezawa S, Takahashi M, Ohi M, et al. Clinical Significance of Adjuvant Surgical Resection for Initially Unresectable Pancreatic Cancer Responsive to Arterial Infusion Chemotherapy. Hepatogastroenterology. 2014;61:828-33.

8. DeNittis AS, Stambaugh MD, Lang P, Wallner PE, Lustig RA, Dillman RO, et al. Complete remission of nonresectable pancreatic cancer after infusional colloidal phosphorus-32 brachytherapy, external beam radiation therapy, and 5-fluorouracil: a preliminary report. Am J Clin Oncol. 1999;22:355-60.

9. Jin Z, Du Y, Li Z, Jiang Y, Chen J, Liu Y. Endoscopic ultrasonography-guided interstitial implantation of iodine 125-seeds combined with chemotherapy in the treatment of unresectable pancreatic carcinoma: a prospective pilot study. Endoscopy. 2008;40:314-20.

10. Popescu CC, Wise J, Sowards K, Meigooni AS, Ibbott GS. Dosimetric characteristics of the Pharma Seed model BT-125-I source. Med Phys. 2000;27:2174-81.

11. Yu YP, Yu Q, Guo JM, Jiang HT, Di XY, Zhu Y. (125)I particle implantation combined with chemoradiotherapy to treat advanced pancreatic cancer. Br J Radiol. 2014;87:20130641.

12. Zou YP, Li WM, Zheng F, Li FC, Huang H, Du JD, et al. Intraoperative radiofrequency ablation combined with 125 iodine seed implantation for unresectable pancreatic cancer. World $\mathrm{J}$ Gastroenterol. 2010;16:5104-10.

13. Hong GB, Zhou JX, Sun HB, Li CY, Song LQ. Continuous transarterial infusion chemotherapy with gemcitabine and 5-Fluorouracil for advanced pancreatic carcinoma. Asian Pac J Cancer Prev. 2012;13:2669-73.

14. Montemaggi P, Dobelbower R, Crucitti F, Morganti AG, Smaniotto D, Luzi S, et al. Interstitial brachytherapy for pancreatic cancer: report of seven cases treated with $125 \mathrm{I}$ and a review of the literature. Int J Radiat Oncol Biol Phys. 1991;21:451-7.

15. Wang ZM, Liu Y, Liu FJ, Chen KM, Huang G. Clinical efficacy of CT-guided iodine-125 seed implantation therapy in patients with advanced pancreatic cancer. Eur Radiol. 2010;20:1786-91.

16. Xu KC, Niu LZ, Hu YZ, He WB, He YS, Li YF, et al. A pilot study on combination of cryosurgery and (125)iodine seed implantation for treatment of locally advanced pancreatic cancer. World $\mathrm{J}$ Gastroenterol. 2008;14:1603-11.

17. Jiang FQ, Lu W, Yang C, Du P, Ma JP, Yang J, et al. Curative effect of transcatheter arterial chemoembolization combined with radiofrequency ablation in treating hepatic cell carcinoma and its effect on serum markers. Cancer Biomark. 2017;20:17-22.

18. Kim JW, Shin SS, Kim JK, Choi SK, Heo SH, Lim HS, et al. Radiofrequency ablation combined with transcatheter arterial chemoembolization for the treatment of single hepatocellular carcinoma of 2 to 
$5 \mathrm{~cm}$ in diameter: comparison with surgical resection. Korean J Radiol. 2013;14:626-35.

19. Yin X, Zhang L, Wang YH, Zhang BH, Gan YH, Ge NL, et al. Transcatheter arterial chemoembolization combined with radiofrequency ablation delays tumor progression and prolongs overall survival in patients with intermediate (BCLC B) hepatocellular carcinoma. BMC Cancer. 2014;14:849.

20. Sun H, Zhang M, Liu R, Liu Y, Hou YW, Wu CJ. Endovascular implantation of (125)I seed combined with transcatheter arterial chemoembolization for unresectable hepatocellular carcinoma. Future Oncol 2018.

Figures

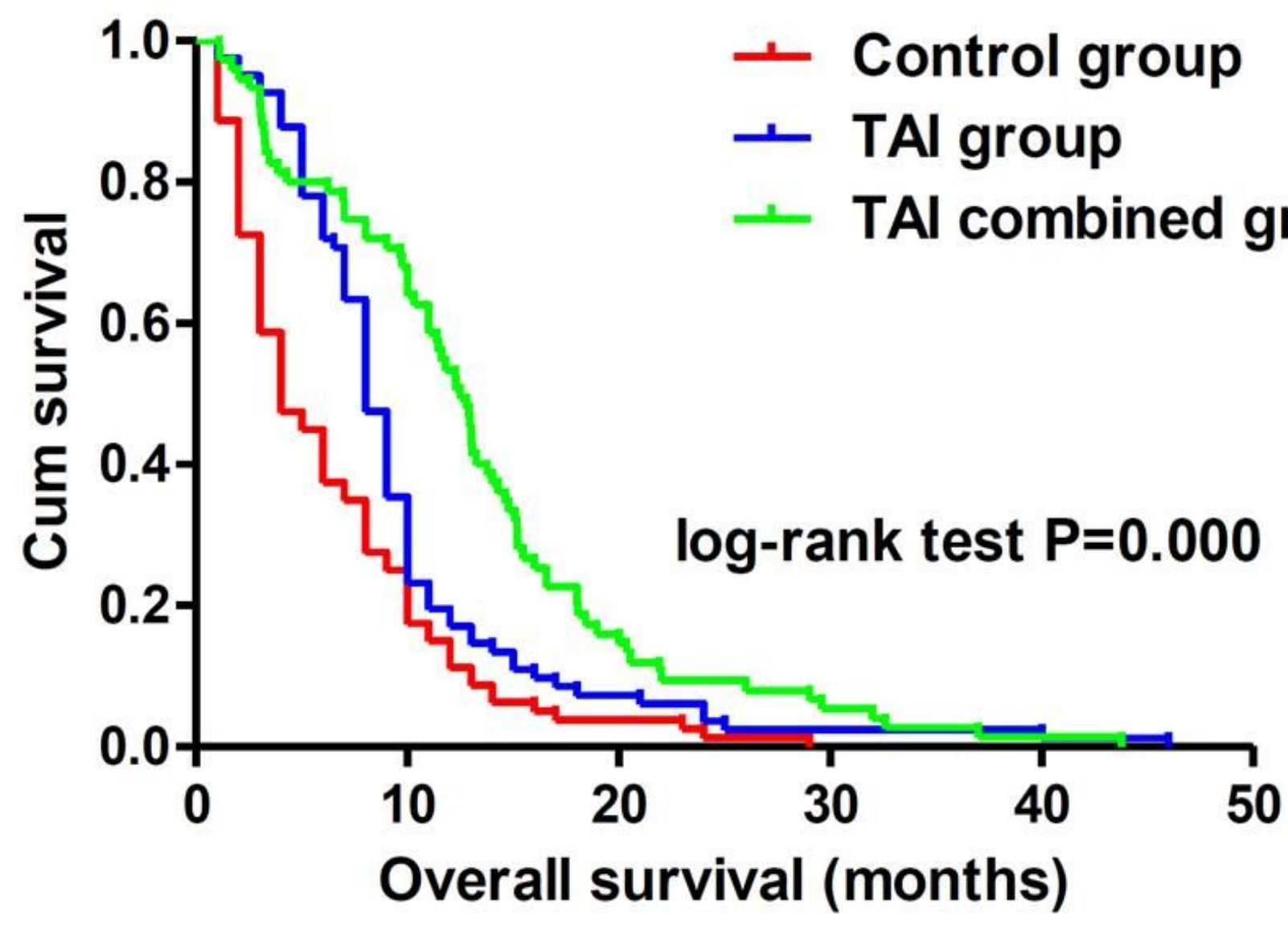

Figure 1

Kaplan-Meier estimates of the overall survival rates among the three groups (control, TAl, and the TAI combined group) 

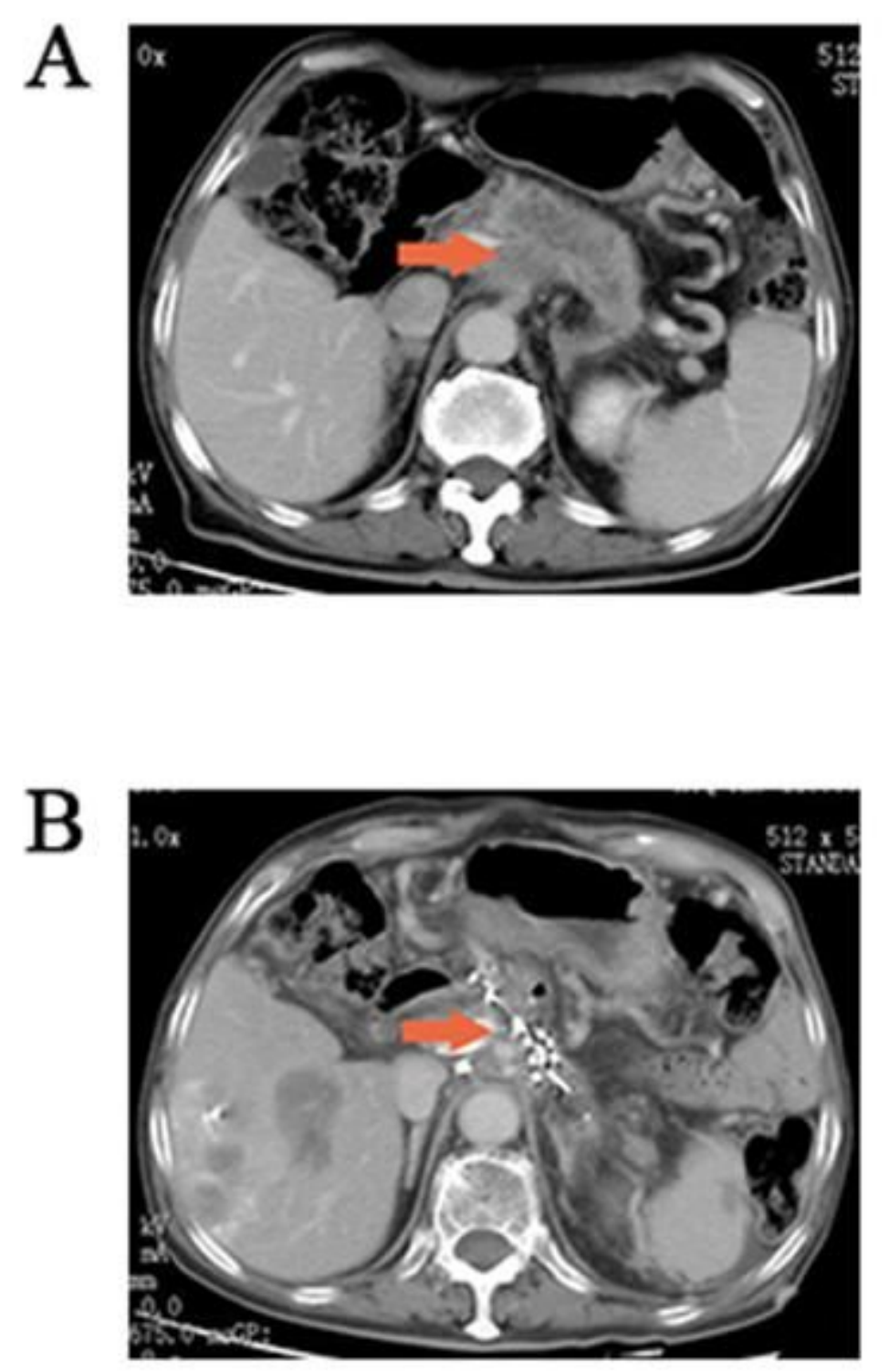

\section{Figure 2}

Representative CT images of one case studie treated with arterial infusion combined with radioactive particles therapy A and B: A. The cancer was located in the pancreatic body (arrow). B. After arterial infusion combined with radioactive particles therapy, a partial response was achieved (arrow). 